\title{
PROCESOS DE SIMPLIFICACIÓN FONOLÓGICA EN NIÑOS CON FISURA LABIOVELOPALATINA INTERVENIDOS QUIRÚRGICAMENTE
}

\author{
Phonological processes in children \\ with surgery treated cleft lip and palate
}

\author{
Pía Villanueva Bianchini (1), María Angélica Fernández Gallardo (2), \\ Maria Loreto Lizana Sánchez ${ }^{(3)}$, Hernán M. Palomino ${ }^{(4)}$
}

\begin{abstract}
RESUMEN
Objetivo: conocer las características del desempeño fonológico en niños con fisura labiovelopalatina uni y bilateral, entre 3 y 4,11 años. Método: se evaluaron 39 pacientes con fisura labiovelopalatina tratada quirúrgicamente, los cuales fueron divididos en 4 grupos de estudio, según el tipo de fisura (uni o bilateral) y edad (3-3,11 años y 4-4,11 años). Para la determinación de la cantidad, tipo y frecuencia de procesos de simplificación fonológica en el grupo de estudio, se aplicó el Test de Procesos de simplificación fonológica (Chile). Los puntajes obtenidos fueron comparados con la norma a través del análisis estadístico t test y analizados descriptivamente. Resultados: se observó una cantidad significativamente mayor de procesos fonológicos presentes en niños con fisura respecto a la norma. Para todos los grupos de estudio los procesos fonológicos de simplificación más frecuentes fueron los de sustitución, con excepción del grupo de niños con fisura unilateral de 3-3,11 años, donde los procesos más frecuentes fueron los relativos a la estructura silábica. Conclusiones: los resultados obtenidos sugieren la necesidad de incluir técnicas de evaluación de la presencia de procesos fonológicos en niños fisurados con el fin que las terapias consideren el entrenamiento para la eliminación de estos procesos en etapas adecuadas del desarrollo, con el fin de mejorar el aspecto conversacional del lenguaje.
\end{abstract}

DESCRITORES: Desarrollo del Lenguaje; Labio Leporino; Fisura del Paladar; Transtornos de la Articulación

\section{INTRODUCCIÓN}

La comunicación oral de los niños nacidos con fisura labiovelopalatina es una de las funciones

(1) Fonoaudióloga; Profesor Asistente na Universidad de Chile, Chile; Magíster en Ciencias Odontológicas por la Universidad de Chile; Especialista en Motricidad Orofacial por CEFAC - Pós-Graduação em Saúde e Educação.

(2) Fonoaudióloga; Académico Instructor Universidad de Chile; Magíster en Fisiopatología Craneocervical, Craneomandibular y Dolor Facial por Universidad Andrés Bello, Santiago, Chile.

(3) Cirujano Dentista; Práctica privada; Licenciado en Odontología por la Universidad de Chile.

(4) Cirujano Dentista; Profesor Asistente Universidad de Chile; Profesor Titular Universidad Andrés Bello, Santiago, Chile; Especialista en Ortodoncia por la Universidad de Chile; Magíster en Odontología.

Conflito de interesses: inexistente que se observa generalmente alterada, tanto en sus características fonoarticulatorias como en el lenguaje, que requieren terapias fonoaudiológicas específicas ${ }^{1,2}$. Respecto a este último proceso, se ha observado que los pacientes con fisura presentan un menor desarrollo del lenguaje, principalmente del lenguaje expresivo ${ }^{3}$ y que existe un estilo menos asertivo de participación en la conversación que puede persistir durante toda la infancia ${ }^{1,4}$.

La mayoría de los estudios coinciden en que dentro de las variadas causas para el retraso en el desarrollo del lenguaje, la principal sería la alta prevalencia de enfermedad en el oído medio ${ }^{5-7}$.

Dentro de las alteraciones fonoaudiológicas observadas en pacientes con fisura, se ha encontrado una mayor utilización de los procesos de 
simplificación fonológica, especialmente a las edades de 3 a 4 años ${ }^{8}$. Estos procesos corresponden a procedimientos realizados por los niños con el fin de simplificar la producción verbal, considerándose normales hasta cierta edad, ya que luego se van eliminando con el desarrollo fonológico hasta lograr la emisión de la palabra según un modelo adulto, lo que ocurre alrededor de los 6 años ${ }^{9}$. El desarrollo de estos procesos fonológicos, se ha visto interrelacionado con el desarrollo de la memoria de trabajo, de la memoria fonológica y del deletreo, los que a su vez se relacionan con la edad cronológica, el crecimiento y desarrollo, y el grado de escolaridad ${ }^{10,11}$.

Estos procesos han sido clasificados por Ingram ${ }^{12}$ en tres tipos: los procesos relativos a la estructura silábica, que simplifican la palabra mediante la fusión y/o la omisión de sus sílabas; los procesos de sustitución, que implican la sustitución de fonemas por otros que no están presentes en la palabra y los procesos de asimilación que consisten en asemejar fonemas a otros presentes en la palabras. Dentro de cada uno de estos procesos existen diferentes subtipos, entre los cuales se encuentran, dentro de los procesos de estructura silábica: simplificación de grupo consonántico, omisión de sílaba pretónica, omisión de consonante inicial. Dentro de los procesos de sustitución se encuentran la aspiración de trabantes, posteriorización, nasalización de trabantes y nasalización de consonantes. Finalmente dentro de los procesos de asimilación se observa la asimilación nasal, la asimilación velar y asimilación de sílabas.

El retraso en la supresión de los procesos fonológicos, corresponde a una alteración que se ha visto asociada a diferentes estados, por ejemplo nacimiento prematuro ${ }^{13}$, al alto riesgo de presentar patología auditiva ${ }^{14,15}$ y a patologías como el Síndrome de Down ${ }^{16}$. Aún cuando existen pocos estudios acerca de las alteraciones fonológicas en los niños fisurados, estos han demostrado que en estos pacientes existe un retraso en la supresión de los procesos de simplificación fonológica respecto a los niños de la misma edad sin fisura ${ }^{1,8}$. Es por esto, que los pacientes con presencia de fisura labiovelopalatina, exigen un tratamiento precoz y multidisciplinario, en el cual no solo se reconozcan las alteraciones del habla, más frecuentemente descritas, sino también las alteraciones del lenguaje o del retraso en su desarrollo, con el fin de instaurar terapias que logren objetivos específicos, como la supresión de los procesos de simplificación en la edad adecuada ${ }^{17}$.

El objetivo del presente estudio es conocer la cantidad de procesos de simplificación fonológica (PSF) presentes en niños chilenos con fisura labiovelopalatina de entre 3 y 4 años 11 meses, y compararlos con la norma chilena. Así como conocer la distribución de tipos y subtipos de PSF en pacientes con fisura labiovelopalatina uni $y$ bilateral.

\section{MÉTODO}

Para lograr el objetivo de este trabajo, se diseñó un estudio descriptivo comparativo.

Para conformar el grupo de estudio, se seleccionaron 39 niños, cuyas edades se encontraban en el rango entre 3 años y 4 años 11 meses de edad, con presencia de fisura labiovelopalatina uni y bilateral. Los niños se encontraban en tratamiento fonoaudiológico por alteraciones del habla en un hospital y una fundación orientadas a la terapia de estos pacientes, en la ciudad de Santiago, Chile.

Los sujetos debían cumplir con los siguientes criterios de inclusión: fisura labiovelopalatina tratada quirúrgicamente, niveles de audición dentro de rangos normales. Los sujetos que presentaban antecedentes de fisura sindróminca, así como trastornos cognitivos, motores y/o sensoriales asociados no fueron incluidos en este estudio. Los datos fueron obtenidos a través de las fichas personales y del diagnóstico de los especialistas tratantes.

De esta manera se constituyeron 4 grupos, según tipo de fisura y edad:

- Grupo 1 ( $n=15)$ : niños con fisura labiovelopalatina unilateral de 3 años a 3 años 11 meses.

- Grupo 2 ( $n=11)$ : niños con fisura labiovelopalatina unilateral de 4 años a 4 años 11 meses.

- Grupo $3(n=5)$ : niños con fisura labiovelopalatina bilateral de 3 años a 3 años 11 meses.

- Grupo $4(n=8)$ : niños con fisura labiovelopalatina bilateral de 4 años a 4 años 11 meses.

Todos los sujetos fueron evaluados a través del test de articulación a repetición (TAR) ${ }^{18}$, con el fin de detectar dificultades articulatorias.

Los procedimientos de recolección e interpretación de datos de esta investigación fueron aprobados por el Comité de Ética de la Escuela de Fonoaudiología mediante la resolución número: MO 05/09.

Para la determinación de la cantidad, tipo y frecuencia de procesos de simplificación fonológica en el grupo de estudio, se aplicó el Test de Procesos de simplificación fonológica o TEPROSIF ${ }^{19}$, creado y validado para la población chilena, administrado por un solo operador calibrado (kappa 0.80). El desempeño de los pacientes durante el examen fue grabado y posteriormente analizado por el especialista. 
Los datos obtenidos fueron tabulados y sometidos al análisis estadístico t de student, para realizar la comparación entre grupos de estudio y la norma, y entre los tipos de fisura. La distribución por grupo de PSF y la frecuencia de subtipos de PSF, además de las comparaciones según tipo de fisura y rango etáreo, serán analizadas descriptivamente.

\section{RESULTADOS}

Se obtuvo un puntaje de TEPROSIF para cada niño evaluado, los cuales se promediaron para obtener un puntaje por grupo de estudio. De esta manera el puntaje promedio correspondiente al grupo 1 fue de 66,4, al grupo 2 fue de 47,8, al grupo 3 fue de 68,6 y el correspondiente al grupo 4 fue de 52,5 . Estos puntajes promedio fueron comparados estadísticamente con el puntaje que representa a la norma para el rango etáreo, obteniéndose diferencias significativas para todos los grupos (Tabla 1 y 2 ).
El análisis descriptivo de la distribución de procesos de simplificación (estructura silábica, sustitución, asimilación y otros) arrojó distintos porcentajes para cada uno de los grupos de estudio, los cuales pueden ser observados en la Tabla 3 . Se observa que para todos los grupos de estudios los PSF más frecuentes corresponden a los procesos de sustitución, con excepción del grupo de fisurados unilaterales, entre 3 y 3,11 años, donde los PSF más frecuentes corresponden a los procesos de estructura silábica.

Finalmente, para cada tipo de PSF se obtuvieron los porcentajes de todos los subtipos presentes. De esta manera para el grupo 1 fue posible observar los que los tres subtipos más frecuentes fueron:

Procesos de estructura silábica: omisión de sílaba pretónica $(24,9 \%)$, omisión de consonante inicial $(20,58 \%)$ y omisión de consonante trabante $(19,41 \%)$.

Procesos de sustitución: nasalización de consonantes $(16,45 \%)$, aspiración de trabantes $(15,50 \%)$, lateralización de líquidas vibrantes (10,44\%).

Tabla 1 - Puntaje promedio obtenido en TEPROSIF, según tipo de fisura, en indivíduos entre 3 y 3,11 años y su comparación con la norma

\begin{tabular}{ccccccc}
\hline & Puntaje X & DS & Norma X & DS & t & p $^{*}$ \\
\hline FLVP Unilateral $(\mathbf{n = 1 5})$ & 66,4 & 19,5 & 28,47 & 3,98 & 7,381 & 0,000 \\
FLVP Bilateral $(\mathbf{n = 5 )}$ & 68,6 & 15,4 & 28,47 & 3,98 & 5,641 & 0,000 \\
\hline
\end{tabular}

* Test $t$ de student

Tabla 2 - Puntaje promedio obtenido en TEPROSIF, según tipo de fisura, en indivíduos entre 4 y 4,11 años y su comparación con la norma

\begin{tabular}{ccccccc}
\hline & Puntaje X & DS & Norma X & DS & t & p $^{*}$ \\
\hline FLVP Unilateral $(\mathbf{n = 1 1 )}$ & 47,18 & 17,4 & 12,41 & 4,09 & 6,452 & 0,000 \\
FLVP Bilateral $(\mathbf{n = 8})$ & 52,5 & 25,7 & 12,41 & 4,09 & 4,357 & 0,000 \\
\hline
\end{tabular}

* Test $t$ de student

Tabla 3 - Distribución de procesos de simplificación fonológica (PSF) según tipo de fisura y edad

\begin{tabular}{cccccccccc}
\hline & \multicolumn{2}{c}{$\begin{array}{c}\text { Estructura } \\
\text { Silábica }\end{array}$} & \multicolumn{2}{c}{ Sustitución } & \multicolumn{2}{c}{ Asimilación } & \multicolumn{2}{c}{ Otros } & \multirow{2}{*}{ Total } \\
\cline { 2 - 12 } & № & $\%$ & № & $\%$ & № & $\%$ & № & $\%$ & № \\
\hline FLVP unilateral (3-3,11 años) & 519 & 50 & 314 & 30 & 177 & 17 & 26 & 3 & 1036 \\
FLVP unilateral (4-4,11 años) & 184 & 35 & 258 & 50 & 75 & 14 & 3 & 1 & 520 \\
FLVP bilateral (3-3,11 años) & 137 & 40 & 141 & 42 & 59 & 17 & 5 & 1 & 342 \\
FLVP bilateral (4-4,11 años) & 135 & 32 & 199 & 47 & 87 & 21 & 0 & 0 & 421 \\
\hline TOTAL & 975 & 42,04 & 912 & 39,3 & 398 & 17,16 & 34 & 1,46 & 2319 \\
\hline
\end{tabular}


Procesos de asimilación: asimilación nasal (77,84\%), asimilación dental $(5,11 \%)$, vibratización de líquidas $(4,54 \%)$.

El mismo análisis fue realizado para el resto de los grupos obteniendo los siguientes resultados:

Grupo 2: Procesos de estructura silábica: omisión de sílaba pretónica $(20,94 \%)$, omisión de consonante trabante $(15,70 \%)$, síncopa $(15,18 \%)$.

Procesos de sustitución: aspiración de trabantes $(22,31 \%)$, lateralización de líquidas vibrantes (13,94\%), posteriorización $(9,16 \%)$.

Procesos de asimilación: asimilación nasal $(47,29 \%)$, asimilación dental $(17,56 \%)$, asimilación vocálica $(9,45 \%)$.

Grupo 3: Procesos de estructura silábica: síncopa $(25,73 \%)$, omisión de sílaba pretónica $(22,05 \%)$, omisión de consonante inicial $(20,58 \%)$.

Procesos de sustitución: aspiración de trabantes $(26,42 \%)$, posteriorización $(18,57 \%)$, lateralización de líquidas vibrantes (16,42\%).

Procesos de asimilación: asimilación nasal $(58,30 \%)$, asimilación velar $(11,60 \%)$, asimilación vocálica y dental (5\%).

Grupo 4: Procesos de estructura silábica: omisión de sílaba pretónica $(27,04 \%)$, omisión de consonante trabante $(20,49 \%)$, simplificación de grupo consonántico $(17,21 \%)$.

Procesos de sustitución: aspiración de trabantes (22,92\%), posteriorización (18,04\%), nasalización de consonantes $(11,7 \%)$.

Procesos de asimilación: asimilación nasal $(67,7 \%)$, velar $(6,66 \%)$, vocálica $(5,55 \%)$.

\section{DISCUSIÓN}

En pacientes con fisura labiovelopalatina es posible encontrar una capacidad intelectual normal para el aprendizaje de un sistema de lenguaje complejo. El niño presentará un mayor riesgo de dificultades del habla debido a las alteraciones estructurales o secuelas postquirúrgicas que modificaran el mecanismo de producción de las palabras ${ }^{20}$.

Según los resultados obtenidos en este estudio, se observa una presencia significativamente mayor de procesos de simplificación fonológica en los niños entre 3 y 4,11 años, tanto con fisura unilateral como bilateral, al compararlos con la norma. Según Chapman, los pacientes con y sin fisura de dos años de edad mostrarían un desempeño similar del lenguaje, al comparar los procesos de simplificación fonológica presentes en este grupo, con excepción de los procesos de asimilación nasal y posteriorización de consonantes, que muestran diferencias significativas pero cuyos bajos porcentajes de utilización los hacen de poca importancia clínica ${ }^{21}$. En esta investigación se observó que en todos los grupos de estudio el proceso de asimilación más frecuente correspondía a la asimilación nasal, lo que sugiere que este proceso de simplificación es más frecuente debido a la incompetencia velofaringea que presentan la mayoría de los niños con fisura labiovelopalatina, traduciéndose en hipernasalidad y emisión nasal al momento de hablar ${ }^{1}$.

En 1993, Chapman observó al comparar, en este caso, niños de 3, 4 y 5 años de edad con y sin fisura, que las diferencias significativas en el número de procesos utilizados se observan durante los 3 y 4 años, mientras que las diferencias en el tipo de procesos utilizados por separado se observan solo a los 3 años. Esto sugiere que existe un retraso temprano en la eliminación de los procesos de simplificación fonológica, el cual puede solucionarse de manera espontánea, aún cuando persiste un número de niños que mantienen los procesos más allá de los 5 años ${ }^{8}$.

Según Morris, dentro de un grupo de niños fisurados es posible encontrar dos subgrupos, a la edad de dos años: un subgrupo con desarrollo normal del lenguaje y otro con un retraso en el desarrollo del lenguaje expresivo. Al comparar entre estos subgrupos, a la edad de 3 años, es posible observar diferencias significativas en la utilización de los siguientes procesos de simplificación: simplificación de grupo consonántico, omisión de consonante final, nasalización de consonantes, utilización de fonemas oclusivos o fricativos glóticos, sustitución del fonema interdental fricativo por el fonema labiodental fricativo y omisión de consonante medial, los cuales se presentan en un mayor porcentaje en el subgrupo cuyo lenguaje expresivo se encontraba retrasado. Esto sugiere que el tratamiento de este retraso permitiría una mejoría temprana del lenguaje, evitando el porcentaje remanente de pacientes que no muestran una resolución espontánea de las alteraciones a los 5 años ${ }^{22}$.

Los resultados de esta investigación sugieren que, además de la asimilación nasal, los procesos de simplificación fonológica más frecuentes en todos los grupos estudiados corresponden a la omisión de sílaba pretónica, que es un proceso que afecta la estructura de la sílaba, y la aspiración de trabantes, un proceso de sustitución, lo que es diferente a lo reportado por Morris, quienes obtuvieron mayores frecuencias en otros procesos de simplificación fonológica de estructura silábica. Por otro lado, es importante considerar que se ha visto que en las diferentes edades de un niño no se observan diferencias significativas entre los macroprocesos 
(estructura silábica, sustitución, asimilación), lo que explica el hecho de que las comparaciones sean realizadas sólo con los subgrupos de cada proceso ${ }^{23}$.

Los resultados obtenidos por Morris sugieren la necesidad de la implementación de técnicas de evaluación de la presencia de procesos fonológicos, de manera que las terapias abarquen no sólo las alteraciones fonéticas presentes, si no también el entrenamiento para la eliminación de estos procesos en etapas adecuadas del desarrollo, con el fin de mejorar el aspecto conversacional del lenguaje ${ }^{22}$.

Algunos autores han demostrado que el tratamiento con ortopedia en la infancia permite que en edades tempranas se encuentren menos alteraciones en el desarrollo fonológico. En el caso del presente estudio, los niños con fisura no fueron tratados con ortopedia, lo que podría explicar la alta utilización de PSF en todos los pacientes de los grupos de estudio. Según Konst et al, al evaluar las diferencias en el desarrollo fonológico en pacientes con fisura unilateral, con y sin tratamiento de ortopedia temprana, se encontraron diferencias significativas a la edad de 2, 2 1/2, 3 y 6 años, donde el primer grupo mostró un desarrollo fonológico retrasado o normal en comparación al segundo grupo que mostró un patrón de desarrollo anormal. Sin embargo, este efecto positivo en el desarrollo del lenguaje sería sólo temprano en el tiempo, ya que finalmente a la edad de 6 años, los niños sin ortopedia alcanzarían el mismo nivel en el patrón del lenguaje expresivo que los niños con tratamiento de ortopedia temprana ${ }^{24}$. Al considerar otras patologías, como por ejemplo, desórdenes respiratorios obstructivos del sueño, también se ha observado que la instauración de una terapia temprana, en este caso tonsilectomía, ayudaría a mejorar el desarrollo de los procesos fonológicos en el niño ${ }^{25}$. De la misma manera, en niños con pérdida de la audición y experiencia temprana en implante coclear, no se observan diferencias fonológicas respecto a sus pares sanos, lo que permite su evaluación con los diferentes test estandarizados ${ }^{26}$. De lo anterior se desprende la necesidad de realizar en estos pacientes, una planificación de tratamiento temprano, que permite dar solución a las más variadas alteraciones, que retrasen el desarrollo del lenguaje.

Por otro lado, estudios actuales han observado una mejoría en el desarrollo fonológico de los niños pre-escolares, una vez que comienzan a manejar el proceso lector ${ }^{27}$. Considerando que no existen en los pacientes fisurados, alteraciones cognitivas que impidan el correcto manejo de la lectura, es posible suponer que durante este aprendizaje existirá una mayor tasa de supresión de la alta cantidad de procesos fonológicos observados en este estudio.

\section{CONCLUSION}

Se concluye que los niños con fisura labiovelopalatina uni y bilateral generan mayor cantidad de procesos fonológicos que lo esperado para su edad según la norma chilena, sin que se observe la variable tipo de fisura en el desempeño lingüístico de los pacientes.

\begin{abstract}
Purpose: to determine the phonological skills of Chilean children between 3 years and 4 years 11 months old with unilateral and bilateral cleft lip and palate. Method: a sample of thirty-nine cleft lip and palate children treated with surgery was divided into four different groups according to the type of cleft lip and palate (unilateral and bilateral) and age (3-3,11 years and 4-4,11 years). We were carried out the TEPROSIF test (Chile), in order to determine the amount, type and frequency of simplification phonological processes in the studied group. The scores obtained were compared with the Chilean standard utilizing the $t$ test statistical package. Descriptive statistics were obtained. Results: more phonologic processes were significantly observed in the cleft group. In all groups the most frequently subtype of simplification phonological processes was the replacement processes' group, except for in the 3-3,11 years of age in unilateral cleft, where the most frequently were the syllabic structure processes. Conclusion: results suggest the need to include techniques for assessing the presence of phonological processes in cleft children in order to consider the training for eliminating these processes in their therapies in the appropriate stages of development, aiming at improving the language conversational aspect.
\end{abstract}

KEYWORDS: Language Development; Cleft Lip; Cleft Palate; Articulation Disorders 


\section{REFERÊNCIAS}

1. Nagarajan R, Savitha VH, Subramaniyan B. Communication disorders in individuals with cleft lip and palate: An overview. Indian J Plast Surg 2009;42:S137-43.

2. Pamplona M del C, Ynsunza A, Pérez G, Vergara $S$. Summer school speech therapy for children with cleft palate and labguage disorder. Gac Med Mex. 2009;145:475-9.

3. Priester GH, Goorhuis-Brouwer SM. Speech and language development in toddlers with and without cleft palate. Int J Pediatr Otorhinolaryngol. 2008; 72 : 801-6.

4. Frederickson MS, Chapman KI, Hardin-Jones M. Conversational skills of children with cleft lip and palate: a replication and extension. Cleft Palate Craniofac J. 2006 Mar; 43(2):179-88.

5. Zambonato TC, Feniman MR, Blasca WQ, Lauris JR, Maximino LP. Braz J Otorhinolaryngol. 2009;75:888-92.

6. Ruiter JS, Korsten-Meijer AGW, GoorhuisBrouwer SM. Communicative abilities in toddlers and in early school age children with cleft palate. Int J Pediatr Otorhinolaryngol. 2009; 73:693-8.

7. Vallino LD, Zuker R, Napoli JA. A Study of speech, language, hearing, and dentition in children with cleft lip only. Cleft Palate Craniofac J. 2008 Sept; 45(5): 485-94.

8. Chapman KL. Phonologic processes in children with cleft palate. Cleft Palate Craniofac J. 1993 Jan; 30(1):64-72.

9. Dodd B, Holm A, Hua Z, Crosbie S. Phonological development: a normative study of British English children. Clin Linguist Phonet. 2003; 17(8):617-43.

10. Gindri G, Keske-Soares M, Mota HB. Working memory, phonological awareness and spelling hypothesis. Pro Fono. 2007 Jul-Sep; 19(3):313-22.

11. Rodrigues A, Befi-Lopes DM. Phonological working memory and its relationship with language development in children. Pro-Fono. 2009 Jan-Mar; 21(1):63-8.

12. Ingram D. Trastornos fonológicos en el niño. Barcelona. Editorial Médica-Técnica; 1983.

13. Guarini A, Sansavini A, Fabbri C, Alessandroni R, Faldella G, Karmiloff-Smith A. Reconsidering the

http://dx.doi.org/10.1590/S1516-18462011005000091

RECEBIDO EM: 24/12/2009

ACEITO EM: 13/07/2010

Endereço para correspondência:

Pía Villanueva Bianchini

Independência, 1027

Santiago - Chile

CEP: 8380453

E-mail: piavillahk@gmail.com impact of preterm birth on language outcome. Early Hum Dev. 2009 Oct; 85(10):639-45.

14. Chen JL, Messner AH, Curtin G. Newborn hearing screening in infants with cleft palates. Otol Neurotol. 2008; 29:812-5.

15. D'Mello J, Kumar S. Audiological findings in cleft palate patients attending speech camp. Indian $\mathrm{J}$ Med Res. 2007; 125:777-82.

16. Barnes E, Roberts J, Long SH, Martin GE, Berni MC, Mandulak KC, et al. Phonological accuracy and intelligibility in connected speech of boys with fragile $X$ syndrome or Down syndrome. J Speech Lang Hear Res. 2009 Aug; 52(4):1048-61.

17. Scherer NJ, DÁntonio LL, McGahey H. Early intervention for speech impairment in children with cleft palate. Cleft Palate Craniofac J. 2008; 45:18-31 18. Alfaro S, Barrera J, de Barbieri Z, Maggiolo M. Presentación de instrumentos de uso habitual en fonoaudiología. Ediciones Escuela de Fonoaudiología, Facultad de Medicina, Universidad de Chile. Santiago, Chile, 1998.

19. Maggiolo M, Pavez M. Test para evaluar procesos fonológicos de simplificación (TEPROSIF). Ediciones Escuela de Fonoaudiología, Facultad de Medicina, Universidad de Chile. Santiago, Chile, 2000.

20. Lohmander A, Persson C. A longitudinal study of speech production in Swedish children with unilateral cleft lip and palate and two-stage palatal repair. Cleft Palate Craniofac J. 2008; 45:32-41.

21. Chapman K, Hardin M. Phonetic and phonologic skills of two-year-olds with cleft palate. Cleft Palate Craniofac J. 1992 Sept; 29(5):435- 43.

22. Morris H., Ozanne A. Phonetic, phonological, and language skills of children with a cleft palate. Cleft Palate Craniofac J. 2003 Sept; 40(5):460-70.

23. Acosta V, Ramos V. Procesos fonológicos: evaluación y tratamiento. Rev CEFAC. 2006; 8(1):27-35.

24. Konst E, Rietveld T, Peters H, Huijpers-Jagtman A. Language skills of young children with unilateral cleft lip and palate following infant orthopedics: a randomized clinical trial. Cleft Palate Craniofac J. 2003 Jul; 40(4):356-62.

25. Lundeborg I, McAllister A, Samuelsson C, Ericsson E, Hultcrantz E. Phonological development in children with obstructive sleepdisordered breathing. Clin Linguist Phon. 2009 Oct; 23(10):751-61.

26. Spencer LJ, Tomblin JB. Evaluating phonological processing skills in children with prelingual deafness who use cochlear implants. J Deaf Stud Deaf Educ. 2009 Winter; 14(1):1-21.

27. Mousinho R, Correo J. Linguistic and cognitive skills in readers and nonreaders. Pro-Fono. 2009 Apr-Jun; 21(2):113-8. 